\title{
Spatio-temporal analysis of malaria vector density from baseline through intervention in a high transmission setting
}

Victor A. Alegana ${ }^{1,9^{*}}$, Simon P. Kigozi ${ }^{2,5}$, Joaniter Nankabirwa ${ }^{2,3}$, Emmanuel Arinaitwe ${ }^{2}$, Ruth Kigozi ${ }^{2}$, Henry Mawejje ${ }^{2}$, Maxwell Kilama ${ }^{2}$, Nick W. Ruktanonchai ${ }^{1,9}$, Corrine W. Ruktanonchai ${ }^{1,9}$, Chris Drakeley ${ }^{5}$, Steve W. Lindsay ${ }^{6}$, Bryan Greenhouse ${ }^{4}$, Moses R. Kamya ${ }^{2,3}$, David L. Smith, Peter M. Atkinson ${ }^{1,8,10}$, Grant Dorsey ${ }^{4}$ and Andrew J. Tatem ${ }^{1,9}$

\begin{abstract}
Background: An increase in effective malaria control since 2000 has contributed to a decline in global malaria morbidity and mortality. Knowing when and how existing interventions could be combined to maximise their impact on malaria vectors can provide valuable information for national malaria control programs in different malaria endemic settings. Here, we assess the effect of indoor residual spraying on malaria vector densities in a high malaria endemic setting in eastern Uganda as part of a cohort study where the use of long-lasting insecticidal nets (LLINs) was high.

Methods: Anopheles mosquitoes were sampled monthly using CDC light traps in 107 households selected randomly. Information on the use of malaria interventions in households was also gathered and recorded via a questionnaire. A Bayesian spatio-temporal model was then used to estimate mosquito densities adjusting for climatic and ecological variables and interventions.

Results: Anopheles gambiae (sensu lato) were most abundant $(89.1 \% ; n=119,008)$ compared to An. funestus (sensu lato) $(10.1 \%, n=13,529)$. Modelling results suggest that the addition of indoor residual spraying (bendiocarb) in an area with high coverage of permethrin-impregnated LLINs (99\%) was associated with a major decrease in mosquito vector densities. The impact on An. funestus (s.l.) (Rate Ratio 0.1508; 97.5\% Cl: 0.0144-0.8495) was twice as great as for An. gambiae (s.l.) (RR 0.5941; 97.5\% Cl: 0.1432-0.8577).
\end{abstract}

Conclusions: High coverage of active ingredients on walls depressed vector populations in intense malaria transmission settings. Sustained use of combined interventions would have a long-term impact on mosquito densities, limiting infectious biting.

Keywords: Anopheles, Anopheles gambiae, Indoor Residual Spraying, Malaria, Modelling

\section{Background}

Since 2000, there has been a substantial decline in malaria morbidity and mortality globally [1], with prevalence of malaria infections in sub-Saharan Africa (SSA) dropping by a half, associated with the large-scale deployment of long-lasting insecticidal nets (LLINs),

\footnotetext{
* Correspondence: V.A.Alegana@soton.ac.uk; aleganav@gmail.com ${ }^{1}$ WorldPop, Geography and Environment, University of Southampton, Southampton, UK

${ }^{9}$ Flowminder Foundation, Stockholm, Sweden

Full list of author information is available at the end of the article
}

indoor residual spraying (IRS), and prompt and effective treatment with antimalarials [2, 3]. However, SSA still bears the largest Plasmodium falciparum malaria burden and malaria remains a public health problem [4]. This is partly because SSA has the most efficient malaria vector species [5, 6] namely; Anopheles gambiae (s.s.), An. arabiensis and other members of the $A n$. gambiae complex; and An. funestus (s.l.) [7]. It is known that these vectors occur in sympatry across SSA [6]. They are all highly anthropophilic and prefer indoor biting [8]. Anopheles arabiensis, however, tends to 
be less endophagic, yet efficient enough to sustain transmission even if the other species were absent [9]. Vector control is central to the Global Technical Strategy (GTS) for malaria adopted in 2015 by the Global Malaria Programme (GMP) of the World Health Organization (WHO) [10]. Many countries are therefore adding indoor residual spraying (IRS) to the scale up of long lasting insecticidal nets (LLINs) for P. falciparum control $[1,11]$. However, it remains unclear when and how best to combine IRS with LLINs [12], especially given an increasing documentation of pyrethroid resistance $[13,14]$.

There are a limited number of field-based studies assessing the combined impact of interventions on malaria vectors. Cluster randomized trial studies in Tanzania [15], and Benin [16] comparing IRS with bendiocarb plus LLINs versus LLINs alone remain inconclusive. The Tanzania study showed protective effect of IRS when combined with use of insecticide-treated nets (ITNs) compared to ITNs alone; however, the use of ITNs in the study population ranged from $53 \%$ at the start of the survey and declined to $36 \%$. No additional protection was observed in the Benin study and also The Gambia trial [17], which compared LLINs to LLINs plus IRS with dichlorodiphenyltrichloroethane (DDT). Mathematical modelling techniques remain sensitive to the parameters used in the model $[18,19]$. Observational non-randomised studies have also been inconclusive in terms of morbidity and mortality [20-24], and in most cases, it is difficult to assess the direct impact of adding IRS when LLIN or ITN use has not been scaled up in study populations. A multi-country analysis based on national representative household surveys from demographic health surveys (DHS) and malaria indicator surveys (MIS) conducted in 17 SSA countries reported mixed outcomes [20]. The use of combined intervention was protective for medium parasite rate in children 2 to 10 years old, (PfPR $2-10$ between 5 and $40 \%$ ) and high malaria transmission settings $\left(\mathrm{PfPR}_{2-10}\right.$ greater than $40 \%$ ) but not for low transmission settings (less than $\left.5 \% \mathrm{PfPR}_{2-10}\right)$. Given the heterogeneous distribution of mosquitoes, a longitudinal analysis based on field data could reveal how combinations of IRS and LLINs could impact malaria vectors.

Here, we build a geostatistical framework to estimate the spatial and temporal distribution and abundance of primary vectors as part of a longitudinal study in Nagongera sub-county, eastern Uganda from October 2011 to December 2015. The main objectives were to inform vector control strategies by investigating the direct effect of government initiated IRS on malaria vector species. Secondary objectives involved investigating their contemporary distribution and identifying extrinsic abiotic constraints (environmental and ecological covariates) associated with mosquito abundance.

\section{Methods}

\section{Study area}

The study was conducted in an extremely high stable malaria transmission intensity region of Eastern Uganda, south eastern border with Kenya, in Nagongera subcounty [25]. The area has an average altitude of $1,095 \mathrm{~m}$ above sea level and is dominated by subsistence farming (banana, maize and rice). The valleys are drained by east-west flowing streams joining the main river flowing to Lake Kyoga in central Uganda. The area in general experiences two rainy seasons averaging $1000-1500 \mathrm{~mm}$ of rainfall annually. The first wet and longer season is experienced early in the year between March to May and a shorter wet season is from October to November. The average day temperature is approximately $23{ }^{\circ} \mathrm{C}$ with the hottest months being January and February. Malaria transmission is characterised by two main peaks from March to June and November to December. The main malaria vectors in this area are the An. gambiae complex and the An. funestus complex [26, 27]. Full description of the study site and a map, is provided in Additional file 1.

\section{Entomology survey data}

Entomological surveillance was conducted at household level as part of a cohort study described elsewhere $[28,29]$. Briefly, a sampling frame of all the households in the area was established and 100 households selected randomly as part of dynamic cohort study targeting children aged between 6 months and 10 years. The first round of enrolment of households was conducted in August and September 2011, but, households could be dropped from the cohort if individuals moved out of the area. Thus, seven new households were selected in the second round of enrolment in 2013. Consent was obtained from the head of the household as part of the household survey. Mosquitoes were gathered once a month in selected households using the miniature CDC light traps (Model 512, John W. Hock Company, Gainesville, FL, USA). Traps were positioned at the foot end of the bed, next to a person sleeping under a LLIN with a light source placed approximately one meter above the floor [25]. Traps were placed at $1900 \mathrm{~h}$ and retrieved at $0700 \mathrm{~h}$ the following day. Most of the adult female Anopheles mosquitoes were identified using morphological characteristics (95\%) with remaining few identified by polymerase chain reaction (PCR) technique. A comparison of the CDC light trap approach to other methods (i.e. the human-landing catches and pyrethrum spray catches) has already been undertaken elsewhere [25]. A summary of CDC light trap data is shown in section 1 of Additional file 1 and in Additional file 2. 


\section{Climatic and environmental covariates}

Plausible environmental covariates used for modelling vector densities were assembled. These included climatic (rainfall, temperature), ecological (enhanced vegetation index, EVI), topography (elevation), a proxy measure of urbanicity (night-time lights), Euclidean distance of the household to the water sources, and household density (defined as number of households within a $50 \mathrm{~m}$ radius of a selected houses). A generalized linear regression model implemented in the bestglm package in $\mathrm{R}$ was used to check for correlation between covariates that may result in multicollinearity [30]. Covariates (excluding intervention effects) were selected based on Bayesian information criterion (BIC) of most parsimonious nonspatial regression model. Thus, a model with lowest BIC was selected after covariates were regressed against the mosquito counts.

\section{Spatio-temporal analysis of mosquito vector density}

A Bayesian hierarchical generalised mixed model with spatial and temporal effects was used to predict continuous maps of vector densities incorporating the above listed covariates and vector control interventions. An advantage of this approach is the ability to quantify uncertainty in the parameters of interest whilst including missing data points as NAs [31]. Mosquito counts for An. gambiae (s.l.) and An. funestus (s.l.), denoted as $y_{i j} ; i=1, \ldots \ldots, n ; j=1, \ldots \ldots, m$ where $i$ is the household location, and $j$ is the month were modelled as negative binomial [32, 33], with

$$
P\left(Y_{i j}=y_{i j}\right)=\frac{\Gamma\left(k+y_{i j}\right)}{\Gamma(k) \Gamma y_{i j} !} p^{k}(1-p)^{y_{i j}}
$$

Where $\Gamma(\cdot)$ is a gamma function, with dispersion parameter $k$, and variance $\operatorname{var}\left(y_{i j}\right)=\mu_{i j}+\mu_{i j}^{2} / k$ for mean $\mu_{i j}$. The outcome (mean vector density per household per night) for the general mixed effect regression model was of the form

$$
\begin{aligned}
y\left(s_{i}, t_{j}\right)= & \beta_{0}+x^{T}\left(s_{i}, t_{j}\right) \beta_{i}+z_{i}^{T} \gamma_{j} \\
& +\operatorname{Season}\left(\text { month }_{j}\right)+v\left(s_{i}, t_{j}\right)
\end{aligned}
$$

where $x^{T}\left(s_{i}, t_{j}\right) \beta_{i}$ represented several set of covariate effects with $\beta_{i}$ coefficients, $\beta_{0}$ as intercept, $Z_{i}^{T} \gamma_{j}$ representing the random effects, and the last term $v_{i}$ representing the spatial and temporal effect. Binary variables were included for each round of IRS at the household level. The government IRS campaign, using carbamate bendiocarb, was first conducted in the study area between December 2014 and February 2015 (round 1) followed by two rounds in June-July and December 2015. The IRS program was part of a national campaign that started in 2006 in epidemic regions (south western Uganda). In
2009, this was expanded to a further 10 districts in the northern parts of the country and to 14 high burden districts covering the mid-north, north east, mid-eastern and east central in 2014 including the study district (Tororo) [34]. The proportion of individuals sleeping under LLIN was included as a continuous variable. LLINs had been handed out to participating households at the start of the study in 2011 and through government mass campaigns in November 2013. A temporal, independent effect of month was included and modelled as an autoregressive process of first order $\xi_{i j} \sim$ $N\left(0,1 / \tau\left(1-p^{2}\right)\right)$ [35], with initial parameters selected based on a non-spatial time-series first order autoregressive model. Bayesian inference was performed using the integrated nested Laplace approximations (INLA) $[36,37]$ after setting prior distribution to model parameters (the intercept, covariates, spatial and temporal effects, and residual effects). INLA was used because of computational advantages compared to Markov chain Monte Carlo (MCMC) algorithms. This is because Gaussian Markov random fields (GMRFs) are used to represent the continuous domain Gaussian random field (GF) via stochastic partial differential equation (SPDE) approach resulting in computationally efficient matrices. Several plausible models were considered by varying the variable specification, i.e. most complex for all parameters (model 1) to least complex without spatio-temporal effects and interventions (model 5).

Model goodness-of-fits were assessed using a range of parameters including the deviance information criterion (DIC) and the marginal likelihood [38]. Validation was conducted by creating a subset dataset of $n=20$ households selected randomly from the 107 households. Validation statistics included the correlation between the predicted and observed vector densities, and the root mean square error (RMSE). Full model specification details are provided in the supplementary.

\section{Results}

A total of 133,528 adult female Anopheles mosquitoes were collected from 107 houses using CDC light traps between October 2011 and December 2015 (51 months). Of these, An. gambiae (s.l.) [including both An. gambiae (s.s.) and An. arabiensis, hereafter An. gambiae (s.l.)] were most abundant $(119,008 ; 89.1 \%$; 0.3\% fed, followed by An. funestus (s.l.) (hereafter, An. funestus) (13,529; $10.1 \% ; 1.0 \%$ fed). Since An. gambiae (s.s.) and An. arabiensis are not morphologically distinguishable, the proportion of each was examined molecularly and a small percentage $(0.8 \%)$ were $A n$. arabiensis [25]. Mosquito density was modelled by month and the spatio-temporal analysis showed highly heterogeneous and declining vector densities over time, with seasonal peaks twice in a year in May-June and November-December (Fig. 1). 


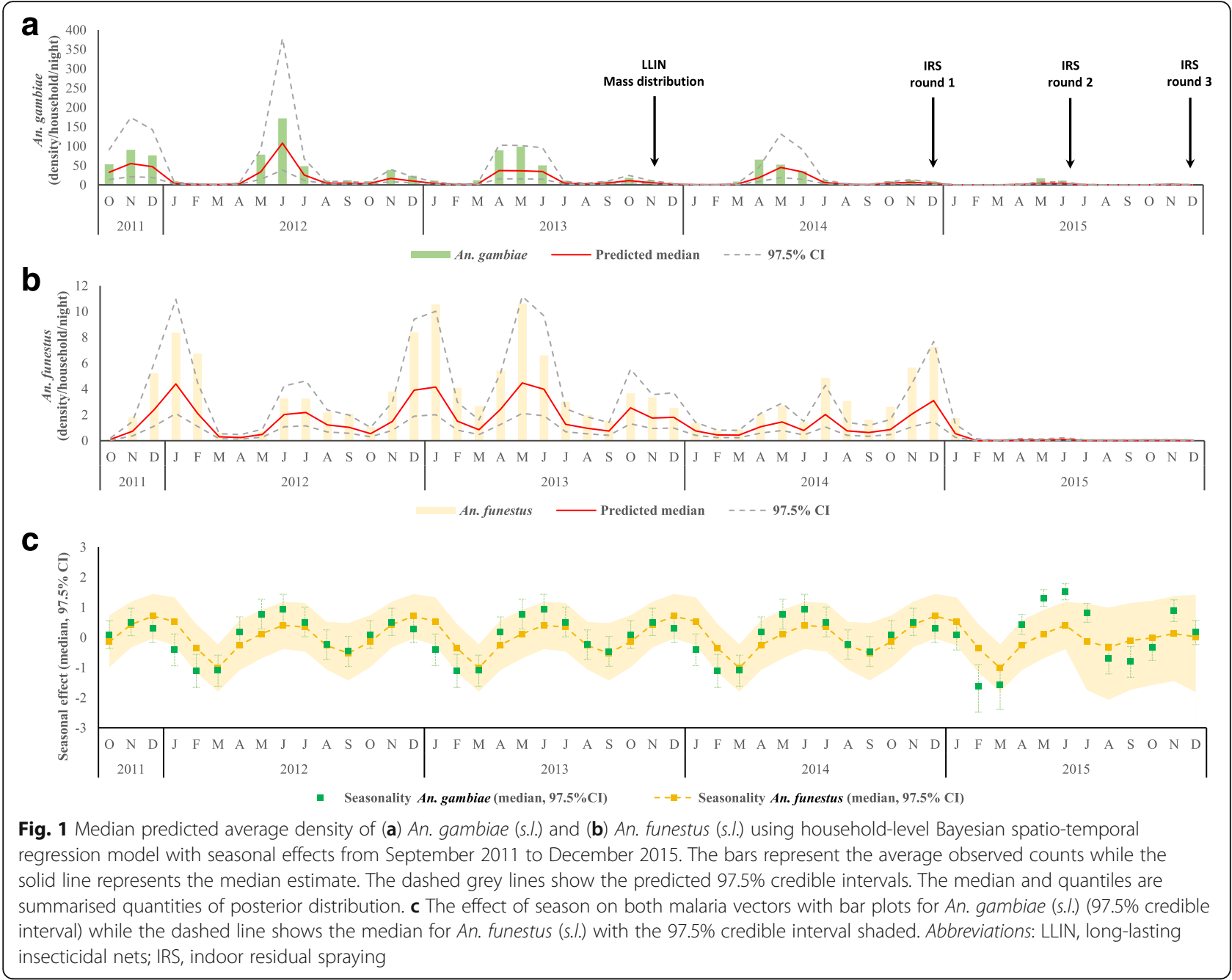

Figure 2 shows the predicted density maps pre-IRS (2011-2014) and post-IRS period (2015). Maps of spatial variance maps are included in the supplementary information.

Table 1 summarises the goodness-of-fit statistics for the five models based on a range of parameters. Comparisons based on combination of the deviance information criterion (DIC) and the marginal likelihood suggested that inclusion of spatial and temporal effects (i.e. models 1-3) improved the models, compared to excluding the spatial effects as in models 4 and 5 . Where two DICs are similar, such as in models 1 and 2, this suggests that little information was gained by the more complex model and extra parametrisation. In terms of spatial parameters of the selected best fitting model (model 1), the spatial range (degree of similarity at household level) was $4 \mathrm{~km}$ for An. gambiae (s.l.) and $2.2 \mathrm{~km}$ for $A n$. funestus. The estimated marginal variance was similar. Model 1 was therefore used for presenting subsequent results.

\section{Effects of environmental and ecological factors on vector counts}

Preliminary regression selected distance to water, elevation, night-time lights, EVI, temperature, rainfall, and household density for An. gambiae (s.l.). Only distance to water, elevation, EVI, rainfall, and household density were selected for An. funestus. EVI was positively correlated with precipitation (Spearman's correlation coefficient of 0.6) but negatively correlated with temperature (Spearman's correlation coefficient of 0.6). Table 2 displays the posterior mean estimates and $97.5 \%$ Bayesian credible intervals for the fixed effect parameters and hyperparameters for the spatio-temporal effect, month, and seasonality. As expected, rainfall and enhanced EVI had a positive (increasing) effect on mosquito densities. Distance to water, on the other hand, had a protective effect $(10.6 \%$ decrease in adult vector abundance for every kilometre increase in distance to water body for An. gambiae (s.l.) and $15.8 \%$ for An. funestus). This is consistent with previous findings on declining mosquito 


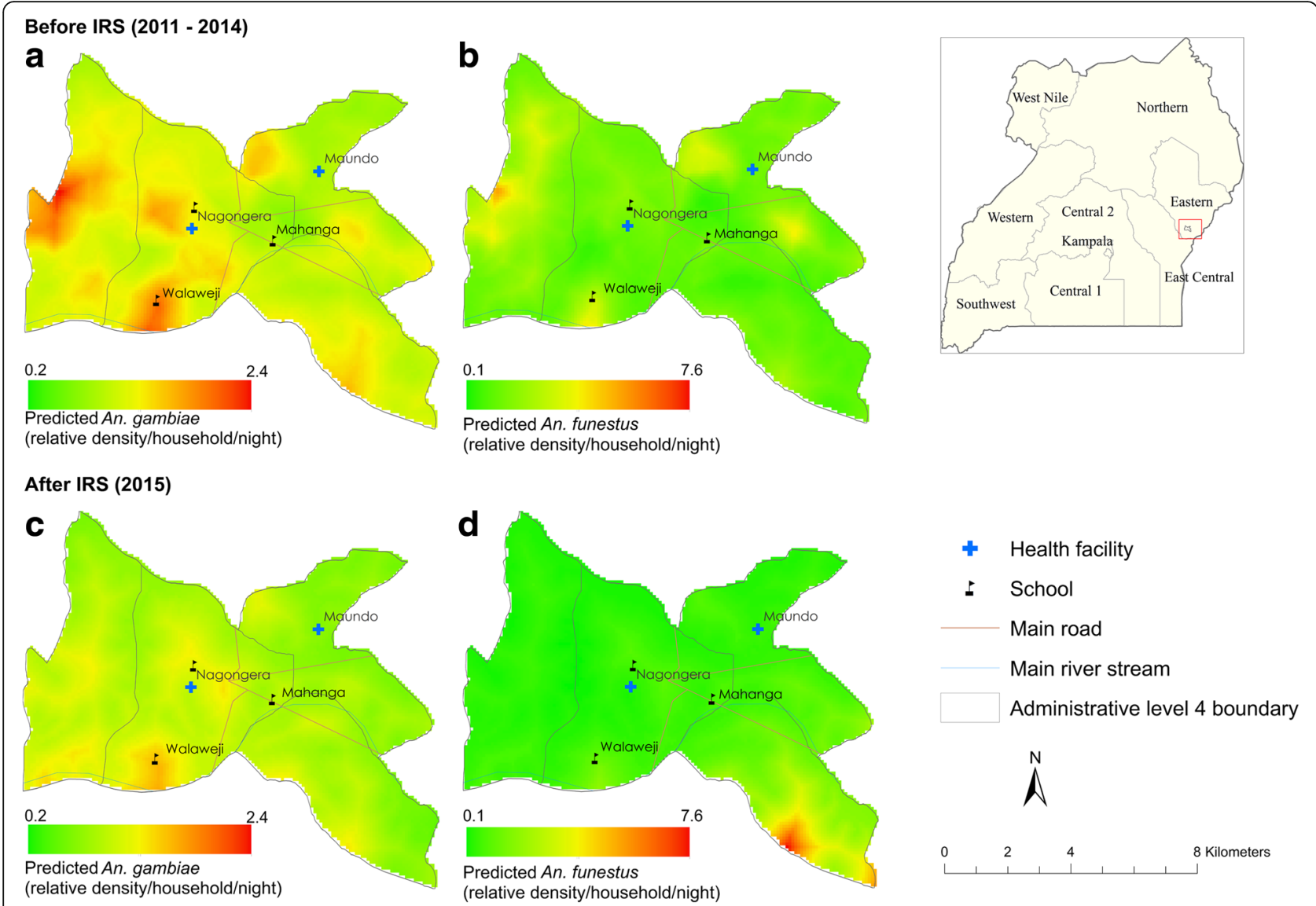

Fig. 2 Map showing the study region in eastern Uganda. The four maps show the average predicted An. gambiae (s.l.) and An. funestus (s.l.) relative density per household per night, respectively, before $(\mathbf{a}, \mathbf{b})$ and after $(\mathbf{c}, \mathbf{d})$ IRS based on the Bayesian spatio-temporal model. The maps are normalised by the mean at $100 \mathrm{~m}$ spatial resolution. Thus, the highest predicted pre-IRS density for An. gambiae (s.l.) was 2.4 times as high as the mean compared to a prediction of 7.6 for An. funestus (s.l.)

Table 1 Model fit and comparison using goodness-of-fit parameters for An. gambiae (s.l.) and An. funestus (s.l.). Model 1 included environmental and climatic variables; random effects (household level and seasonal); intervention use; and spatial and temporal effects. Model 5, the least complex, included only climatic variables and random effects. RMSE and correlation were based on a holdout validation dataset selected randomly $(n=20)$ out of a total 107 households

\begin{tabular}{lllcccc}
\hline Vector species & Model & DIC & Model complexity & Marginal likelihood & RMSE & Correlation (Observed vs Predicted) \\
\hline An. gambiae (s.l.) & Model 1 & 11083.56 & 122.05 & -5743.87 & 1.1059 & 0.7963 \\
& Model 2 & 11080.09 & 119.87 & -5745.77 & 1.0565 & 0.7800 \\
& Model 3 & 11082.78 & 120.42 & -5757.49 & 1.0516 & 0.7777 \\
& Model 4 & 11330.18 & 58.34 & -5838.05 & 1.0883 & 0.7594 \\
& Model 5 & 11329.69 & 56.37 & -5827.67 & 1.0884 & 0.7592 \\
An. funestus (s.l.) & Model 1 & 7188.35 & 134.51 & -3783.64 & 0.9657 & 0.6937 \\
& Model 2 & 7221.12 & 129.62 & -3756.08 & 0.9615 & 0.6984 \\
& Model 3 & 7194.15 & 119.54 & -3764.33 & 0.9172 & 0.6930 \\
& Model 4 & 7385.89 & 51.22 & -3815.50 & 0.9259 & 0.6233 \\
& Model 5 & 7385.90 & 50.89 & -3806.26 & 0.9244 & 0.6252 \\
\hline
\end{tabular}


Table 2 Posterior rate ratio estimates and 97.5\% credible interval (Cl) for the best fitting model (Model 1) for An. gambiae (s.l.) and An. funestus (s.l.). The model includes all data range (2011-2015) and incorporates the effects of interventions. For the spatio-temporal specification, a parameter for the spatial range of influence is shown

\begin{tabular}{|c|c|c|c|c|c|c|c|c|}
\hline \multirow[t]{2}{*}{ Variable } & \multicolumn{4}{|c|}{ An. gambiae (s.l.) Rate Ratio $97.5 \% \mathrm{Cl}$} & \multicolumn{4}{|c|}{ An. funestus (s.l.) Rate Ratio $97.5 \% \mathrm{Cl}$} \\
\hline & Mean & $2.5 \%$ & $50 \%$ & $97.5 \%$ & Mean & $2.5 \%$ & $50 \%$ & $97.5 \%$ \\
\hline Distance to water (estimated in km) & 0.8941 & 0.8520 & 0.8940 & 0.9367 & 0.8421 & 0.7819 & 0.8421 & 0.9064 \\
\hline Elevation (m above sea level) & 0.9805 & 0.9162 & 0.9804 & 1.0520 & 1.0089 & 0.9294 & 1.0089 & 1.0955 \\
\hline Night-time light (intensity) & 0.9749 & 0.9118 & 0.9748 & 1.0420 & - & - & - & - \\
\hline $\mathrm{EVI}$ & 1.1434 & 1.0512 & 1.1433 & 1.2429 & 1.1606 & 1.0150 & 1.1605 & 1.3267 \\
\hline Temperature (estimated in ${ }^{\circ} \mathrm{C}$ ) & 0.9982 & 0.9166 & 0.9981 & 1.0868 & - & - & - & - \\
\hline Precipitation (mm) & 1.0745 & 1.0185 & 1.0745 & 1.1331 & 0.9419 & 0.8438 & 0.9419 & 1.0475 \\
\hline Number of households within $50 \mathrm{~m}$ & 0.9739 & 0.9153 & 0.9739 & 1.0371 & 0.9603 & 0.8885 & 0.9603 & 1.0384 \\
\hline IRS & 0.5941 & 0.1432 & 0.5783 & 0.8577 & 0.1508 & 0.0144 & 0.1472 & 0.8495 \\
\hline LLIN & 1.0026 & 0.9242 & 1.0025 & 1.0884 & 0.9770 & 0.8581 & 0.9769 & 1.1140 \\
\hline Spatial range $(\mathrm{km})$ for Matérn covariance & 4.6341 & 0.2772 & 4.6231 & 5.5876 & 2.2395 & 0.1996 & 2.2284 & 3.7029 \\
\hline
\end{tabular}

Abbreviations: EVI Enhanced vegetation index, IRS Indoor residual spraying, LLIN Long lasting insecticide net

densities with increasing distance from larval sources $[39,40]$. The results for temperature and night-time lights were similar (protective effect) but not important. Day temperature varied between $19{ }^{\circ} \mathrm{C}$ and $40{ }^{\circ} \mathrm{C}$ and previous studies showed that higher temperatures $\left(35^{\circ} \mathrm{C}\right)$ decrease mosquito survival [41].

\section{Temporal effects}

Figure 1 shows the modelled seasonal patterns associated with the two vector species. While the seasonal patterns were similar for the pre-IRS period (2011-2014), the uncertainty increased post-IRS in 2015 for both species. For $A n$. funestus, model predictions showed dampened peaks in 2015 with a large range of uncertainty (the orange band). While similar patterns were predicted for $A n$. gambiae (s.l.) across the years, the range of uncertainty between months increased slightly in 2015. Seasonal peaks and troughs established here provide guidance for targeting IRS before peak transmission [42], but, the dampened effect in 2015 (reduced seasonal peaks) highlights a reduced number of vectors observed at household level after spraying.

\section{Malaria intervention effects}

At the start of the cohort study in 2011, all the enrolled households were provided with LLINs. Over 99\% of cohort study participants reported sleeping under a LLIN the prior evening to the time of routine assessments, done every 3 months [43]. From cross-sectional surveys conducted in the surrounding community (non-cohort households), the proportion of households with at least one LLIN increased from $71.0 \%$ in January 2013 to 95.5\% in January 2015, following a universal LLIN campaign conducted in November 2013. Modelling results show the community-wide deployment of LLINs
(Table 2) did not reduce vector densities. In contrast, the government initiated IRS campaign implemented in December 2014 in the study area with rounds of bendiocarb sprayed approximately every 6 months, had an impact on vector densities. Nationally, IRS campaign in the high burden 14 districts in 2014 and 2015 (including Tororo district) achieved a coverage of 93.5\% [34]. From the cohort, IRS was not done in only 5 houses in the first two rounds of IRS, and in 3 houses in the third round (excluding the 15 houses that dropped from the cohort before round 1 of the IRS). Modelling results showed that IRS was associated with a $40.6 \%$ decrease in mosquito densities for An. gambiae (s.l.), compared to $84.9 \%$ for $A n$. funestus, suggesting a two-fold greater effect on the latter species. Separate bioassays of vector susceptibility in Nagongera conducted in May 2014 showed $100 \%$ mortality rates for bendiocarb but less than $40 \%$ mortality for pyrethroids $24 \mathrm{~h}$ after exposure (unpublished data). This suggests a possible biological mechanism for the additional benefit of bendiocarb in settings with high coverage with pyrethroid-treated LLINs.

\section{Discussion}

Vector control with large-scale deployment of LLINs and IRS are the major methods for malaria control in SSA [1]. However, as countries expand their malaria control programmes, there is a need to assess the impact of combined interventions on malaria vectors as well as health outcomes in routine conditions. The present study focused on entomological outcomes rather than clinical outcomes and the results provide an understanding of seasonal variation in the dominant malaria vectors as well as showing that IRS had an impact on malaria vectors in the setting of high LLIN coverage. 
A longitudinal dataset was used to examine the temporal changes of malaria vector species in a high malaria transmission intensity setting. Such entomological surveillance data are rarely available to assess the impact of IRS on vector densities when there is a high coverage of pyrethroid-treated LLINs. The IRS effect was more evident on An. funestus species compared to An. gambiae (s.l.). While fewer An. gambiae (s.l.) were captured in 2015, IRS campaigns led to an almost total disappearance of An. funestus in the study cohort after two consecutive rounds (data shown in Additional files). Indeed, model estimates of the proportional effect of IRS were twice as high on An. funestus (RR $0.1508,97.5 \% \mathrm{CI}$ : 0.0144-0.8495) compared to An. gambiae (s.l.) (RR 0.5941, 97.5\% CI: 0.1432-0.8577). In addition, An. funestus was only present in south-eastern parts of Nagongera after spraying when compared to the distribution before spraying rounds. This is consistent with other studies showing disproportionate impact of IRS on An. funestus [44]. Along with the quality of spraying (not quantified), the Uganda malaria control programme's policy of conducting IRS after every 6 months, potentially increased its impact on the indoor-biting and resting anopheline mosquitoes in this area.

The results on declining vector density support findings on clinical outcomes elsewhere [28], and are also congruent with other entomological indicators such as the human biting rate [25]. Countries in SSA are changing to the use of carbamates and organophosphates, because of increasing evidence of DDT and pyrethroid resistance [13, 45-47]. One challenge of these insecticides is that they are more expensive than DDT and pyrethroids, which may lead to fewer houses being sprayed. However, it has been shown previously that the use of DDT or pyrethroids for IRS had less impact on morbidity compared to bendiocarb in other parts of Uganda [47, 48]. WHO tube bioassays on the effect of DDT and pyrethroids on An. gambiae (s.l.) in Nagongera showed moderate to high resistance $(68,24$, and $37 \%$ mortality rate on An. gambiae (s.l.) for DDT, deltamethrin, and permethrin, respectively, unpublished data). Elsewhere in SSA, there were mixed results on the use of carbamates and DDT for IRS. For example, IRS with DDT did not offer an additional protective effect in communities with moderate malaria transmission and high coverage of LLINs in a cluster randomized trial in The Gambia where vector susceptibility was high [17]. A different observational study in the same country showed that carbamates and organophosphates were more effective compared to using DDT [45]. Additional empirical evidence from Benin also showed a high efficacy of bendiocarb [49]. On the contrary, there has been some evidence on the resistance of An. gambiae (s.l.) to carbamates particularly in West Africa in Côte d'Ivoire
[50], Mali [46], and Burkina Faso [51]. This supports the need for continuous local monitoring of insecticide resistance and rotations or combinations of insecticides in areas with pyrethroid-treated LLINs as proposed by the WHO [52].

Modelling the seasonal cycles of malaria vectors provides useful information as to when IRS can be targeted for maximum efficacy and provide insights into transmission seasonality. From an operational perspective, the current WHO guidelines propose that the completion of indoor spraying should coincide with the buildup of vector density before the peak transmission season [42]. The modelled seasonality was similar for both vectors with bimodal peaks in June and December. This not only suggests that both species can be targeted for spraying at similar times or months of the year, but also shows that optimal targeting would aim at finishing spraying rounds before these peaks, such that there are fresh deposits of insecticides by May and December, respectively.

Rainfall and EVI were important drivers of vector density and seasonality while increasing distance from water sources was associated with lower mosquito density for both An. gambiae (s.l.) and An. funestus. It is important to use these abiotic factors in modelling adult vector densities as well as for spatial prediction. While past studies have shown that environmental variables are important drivers of seasonal patterns [6], the precise relationships and lag periods of these environmental variables are not well understood or generalizable for different malaria endemic settings [53]. In this study, the time-varying variables (rainfall, EVI and temperature) were not lagged. A separate analysis using a similar model specification but relaxing prior specification for covariates (i.e. using smoothing functions such as second-order random walks on continuous covariates) did not improve the goodness-of-fit. Thus, we opted to use these covariates with fixed prior specification [54]. In employing a longitudinal spatio-temporal analysis, the modelling framework addresses jointly the spatial and temporal correlation between sampled vector species at the household level [55]. A different model formulation, such as one that considers only spatial effects, may lead to different spatial variation and potentially mask the effect of time. Adult mosquito dispersal mechanisms can alter their spatial and temporal distribution substantially [56].

There are some additional caveats. The data used in this methodology focused on indoor biting malaria vectors and outdoor biting was not explored. Besides, the micro level effects of mosquito movement (i.e. indoor or outdoor) were not included in the analysis due to lack of empirical data. Although CDC light traps tend to sample indoor vectors [25], a recent study suggested that even 
the outdoor vectors attempt indoor biting [9]. A major finding by Kilama et al. [25] is the ethical suitability and acceptability of CDC light traps compared to the gold standard human landings or exit traps that can be logistically challenging, may be affected by collector biases, and the former also increases the risk of human infection. More importantly, different sampling strategies for the exophagic vectors should be considered [25], along with biotic interactions (competition and predation). Future studies should also consider an analysis of the influence of changing environment, interactions, and interventions on these exophagic vectors. However, while it is easier to assemble data on the environment, it is rather difficult to assemble longitudinal data on insecticide susceptibility or insecticide residual activity. This limits the form and type of analysis. Nonetheless, use of current environmental factors unearthed important seasonal patterns useful for vector control.

\section{Conclusions}

The study demonstrated that there were major reductions in indoor-biting malaria vectors associated with IRS using bendiocarb when introduced in a community with high coverage and use of LLINs. The maps provide a spatial view of areas that can be targeted by spraying teams, for example, targeting the western parts of Nagongera. In Uganda, results elsewhere suggest that the use of bendiocarb complemented the high LLIN coverage $[47,48]$. While these interventions are being scaled up nationally, insecticide resistance should be monitored continuously by the various national malaria control programmes. It is clear that the combination of vector control tools will not be sufficient to eliminate transmission from this area and that further interventions such as improved housing (ongoing trial in The Gambia [57]) are required to achieve this.

\section{Additional files}

Additional file 1: Description of study site, covariates, and Bayesian geostatistical model. (DOC $1660 \mathrm{~kb}$ )

Additional file 2: Summary of average monthly mosquito counts gathered at household level for the study area. (DOC $80 \mathrm{~kb}$ )

\footnotetext{
Abbreviations

BIC: Bayesian information criterion; CDC: Centre for Disease Control; DDT: Dichlorodiphenyltrichloroethane; DHS: Demographic health surveys; DIC: Deviance information criterion; EVI: Enhanced vegetation index; GF: Gaussian field; GMP: Global Malaria Programme; GMRF: Gaussian Markov random fields; GTS: Global technical strategy; INLA: Integrated nested Laplace approximation; IRS: Indoor residual spraying; LLIN: Long lasting insecticide net; MCMC: Markov chain Monte Carlo; MIS: Malaria indicator surveys; RMSE: Root mean square error; SPDE: Stochastic partial differential equation; SSA: Sub-Saharan Africa; WHO: World Health Organization
}

\section{Acknowledgements}

We would like to thank those in the study community and participants who helped in primary data collection. The authors are also grateful for the technical and administrative support provided by the Infectious Diseases Research Collaboration (IDRC) in Uganda.

\section{Funding}

This work was supported by the National Institutes of Health as part of the International Centres of Excellence in Malaria Research (ICMER) Program for Resistance, Immunology, Surveillance and Modelling of Malaria in Uganda (grant number U19AI089674). AJT is supported by a Wellcome Trust Sustaining Health Grant (grant number 106866/Z/15/Z) and Bill and Melinda Gates Foundation (grant numbers OPP1106427, 1032350, OPP1134076).

\section{Availability of data and material}

Data included as supplementary information. Any additional unpublished data can be made available via request to the corresponding author.

\section{Authors' contributions}

VA, SPK, PMA, DLS, MRK, GD and AJT were responsible for study design, analysis, interpretation, and production of final manuscript. VA, JN, SPK, EA, RK, HM, MK and AJT contributed to data assembly and management, interpretation and production of final manuscript. NWR, CWR, CD, SWL and BG contributed to interpretation and production of final manuscript. All authors read and approved the final manuscript.

\section{Competing interests}

The authors declare that they have no competing interests.

\section{Consent for publication}

Not applicable.

\section{Ethics approval and consent to participate}

For each household survey, written informed consent was obtained from the head of the household prior to conducting surveillance. Additional ethical approval was provided by the Makerere University School of Medicine Research and Ethics Committee, the Uganda National Council for Science, the London School of Hygiene and Tropical Medicine Ethics Committee, the Durham University School of Biological and Biomedical Sciences Ethics Committee, and the University of California, San Francisco Committee on Human Research and the University of Southampton (17263).

\section{Author details}

${ }^{1}$ WorldPop, Geography and Environment, University of Southampton, Southampton, UK. ${ }^{2}$ Infectious Diseases Research Collaboration, Kampala, Uganda. ${ }^{3}$ Department of Medicine, Makerere University College of Health Sciences, Kampala, Uganda. ${ }^{4}$ Department of Medicine, San Francisco General Hospital, University of California, San Francisco, USA. ${ }^{5}$ London School of Hygiene and Tropical Medicine, London, UK. ${ }^{6}$ School of Biological and Biomedical Sciences, Durham University, Durham, UK. Institute for Health Metrics and Evaluation, University of Washington, Seattle, USA. ${ }^{8}$ Faculty of Science and Technology, Lancaster University, Lancaster, UK. ${ }^{9}$ Flowminder Foundation, Stockholm, Sweden. ${ }^{10}$ School of Geography, Archaeology and Palaeoecology, Queen's University Belfast, Belfast BT7 1NN, Northern Ireland, UK.

Received: 20 September 2016 Accepted: 28 November 2016 Published online: 12 December 2016

\section{References}

1. World Health Organization. World Malaria Report 2015. Geneva: World Health organization; 2015.

2. Bhatt S, Weiss DJ, Cameron E, Bisanzio D, Mappin B, Dalrymple U, et al. The effect of malaria control on Plasmodium falciparum in Africa between 2000 and 2015. Nature. 2015;256(7572):207-11.

3. Noor A, Mutheu J, Tatem A, Hay S, Snow R. Insecticide-treated net coverage in Africa: mapping progress in 2000-07. Lancet. 2009;373:58-67.

4. Noor AM, Kinyoki DK, Mundia CW, Kabaria CW, Mutua JW, Alegana VA, et al. The changing risk of Plasmodium falciparum malaria infection in Africa: 2000-10: a spatial and temporal analysis of transmission intensity. Lancet. 2014;383(9930):1739-47. 
5. Hay SI, Sinka ME, Okara RM, Kabaria CW, Mbithi PM, Tago CC, et al. Developing global maps of the dominant Anopheles vectors of human malaria. PLoS Med. 2010;7(2):e1000209.

6. Sinka ME, Bangs MJ, Manguin S, Coetzee M, Mbogo CM, Hemingway J, et al. The dominant Anopheles vectors of human malaria in Africa, Europe and the Middle East: occurrence data, distribution maps and bionomic précis. Parasit Vectors. 2010;3(1):1-34.

7. White GB. Anopheles gambiae complex and disease transmission in Africa. Trans R Soc Trop Med Hyg. 1974;68(4):278-301.

8. Huho B, Briët O, Seyoum A, Sikaala C, Bayoh N, Gimnig J, et al. Consistently high estimates for the proportion of human exposure to malaria vector populations occurring indoors in rural Africa. Int J Epidemiol. 2013;42(1):235-47.

9. Killeen GF, Govella NJ, Lwetoijera DW, Okumu FO. Most outdoor malaria transmission by behaviourally-resistant Anopheles arabiensis is mediated by mosquitoes that have previously been inside houses. Malar J. 2016;15(1):1-10.

10. World Health Organization. Global technical strategy for malaria 2016-2030. Geneva: WHO; 2015

11. Eisele TP, Larsen D, Steketee RW. Protective efficacy of interventions for preventing malaria mortality in children in Plasmodium falciparum endemic areas. Int J Epidemiol. 2010;39 suppl 1:i88-101.

12. World Health Organization. WHO guidance for countries on combining indoor residual spraying and long-lasting insecticidal nets, vol. 2. Geneva: WHO/HTM/GMP/MPAC; 2014

13. Ranson H, Lissenden N. Insecticide Resistance in African Anopheles Mosquitoes: A Worsening Situation that Needs Urgent Action to Maintain Malaria Control. Trends Parasitol. 2016;32(3):187-96.

14. Ranson H, N'Guessan R, Lines J, Moiroux N, Nkuni Z, Corbel V. Pyrethroid resistance in African anopheline mosquitoes: what are the implications for malaria control? Trends Parasitol. 2011;27(2):91-8.

15. West PA, Protopopoff N, Wright A, Kivaju Z, Tigererwa R, Mosha FW, et al. Indoor residual spraying in combination with insecticide-treated nets compared to insecticide-treated nets alone for protection against malaria: a cluster randomised trial in Tanzania. PLoS Med. 2014;11(4):e1001630

16. Corbel V, Akogbeto M, Damien GB, Djenontin A, Chandre F, Rogier C, et al. Combination of malaria vector control interventions in pyrethroid resistance area in Benin: a cluster randomised controlled trial. Lancet Infect Dis. 2012; 12(8):617-26.

17. Pinder M, Jawara M, Jarju LB, Salami K, Jeffries D, Adiamoh M, et al. Efficacy of indoor residual spraying with dichlorodiphenyltrichloroethane against malaria in Gambian communities with high usage of long-lasting insecticidal mosquito nets: a cluster-randomised controlled trial. Lancet. 2015;385(9976):1436-46.

18. Brady OJ, Godfray HC, Tatem AJ, Gething PW, Cohen JM, McKenzie FE, et al. Vectorial capacity and vector control: reconsidering sensitivity to parameters for malaria elimination. Trans R Soc Trop Med Hyg. 2016;110(2):107-17.

19. Chitnis N, Schapira A, Smith T, Steketee R. Comparing the effectiveness of malaria vector-control interventions through a mathematical model. Am J Trop Med Hyg. 2010;83(2):230-40.

20. Fullman N, Burstein R, Lim SS, Medlin C, Gakidou E. Nets, spray or both? The effectiveness of insecticide-treated nets and indoor residual spraying in reducing malaria morbidity and child mortality in sub-Saharan Africa. Malar J. 2013;12:62.

21. Okumu FO, Chipwaza B, Madumla EP, Mbeyela E, Lingamba G, Moore J, et al. Implications of bio-efficacy and persistence of insecticides when indoor residual spraying and long-lasting insecticide nets are combined for malaria prevention. Malar J. 2012;11:378.

22. Kleinschmidt I, Schwabe C, Shiva M, Segura JL, Sima V, Mabunda SJ, Coleman M. Combining indoor residual spraying and insecticide-treated net interventions. Am J Trop Med Hyg. 2009;81(3):519-24.

23. Alba S, Hetzel MW, Nathan R, Alexander M, Lengeler C. Assessing the impact of malaria interventions on morbidity through a community-based surveillance system. Int J Epidemiol. 2011;40(2):405-16.

24. Alba S, Nathan $R$, Schulze A, Mshinda H, Lengeler C. Child mortality patterns in rural Tanzania: an observational study on the impact of malaria control interventions. Int J Epidemiol. 2014;43(1):204-15.

25. Kilama M, Smith DL, Hutchinson R, Kigozi R, Yeka A, Lavoy G, et al. Estimating the annual entomological inoculation rate for Plasmodium falciparum transmitted by Anopheles gambiae s.l. using three sampling methods in three sites in Uganda. Malar J. 2014;13:111.

26. Mawejie HD, Wilding CS, Rippon EJ, Hughes A, Weetman D, Donnelly MJ. Insecticide resistance monitoring of field-collected Anopheles gambiae s.I. populations from Jinja, eastern Uganda, identifies high levels of pyrethroid resistance. Med Vet Entomol. 2013;27(3):276-83.

27. Okello PE, Van Bortel W, Byaruhanga AM, Correwyn A, Roelants P, Talisuna $A$, et al. Variation in malaria transmission intensity in seven sites throughout Uganda. Am J Trop Med Hyg. 2006;75(2):219-25.

28. Kamya MR, Arinaitwe E, Wanzira H, Katureebe A, Barusya C, Kigozi SP, et al. Malaria transmission, infection, and disease at three sites with varied transmission intensity in Uganda: implications for malaria control. Am J Trop Med Hyg. 2015;92(5):903-12

29. Yeka A, Nankabirwa J, Mpimbaza A, Kigozi R, Arinaitwe E, Drakeley C, et al. Factors associated with malaria parasitemia, anemia and serological responses in a spectrum of epidemiological settings in Uganda. PLoS One. 2015;10(3):e0118901.

30. McLeod Al, Xu C. bestglm: Best Subset GLM. 2008.

31. Banerjee S, Carling PB, Gelfand AE. Hierarchical modeling and analysis for spatial data. London: Chapman \& Hall/CRC; 2004.

32. Anscombe FJ. The statistical analysis of insect counts based on the negative binomial distribution. Biometrics. 1949;5(2):165-73.

33. Andersen $\mathrm{F}$. The negative binomial distribution and the sampling of insect populations, Proceedings of the 12th International Congress of Entomology, vol. 395. 1964. p. 395.

34. 34.Uganda Bureau of Statistics (UBOS). ICF International: Uganda Malaria Indicator Survey 2014-15 Kampala. Uganda: UBOS and ICF International; 2015

35. Blangiardo M, Cameletti M, Baio G. Rue Hv: Spatial and spatio-temporal models with R-INLA. Spat Spatiotemporal Epidemiol. 2013;4:33-49.

36. Martins T, Simpson D, lindgren F, Rue H. Bayesian computing with INLA: new features. Trondheim. Norway: Department of Mathematical Sciences, Norwegian University of Science and Technology; 2013. p. 29.

37. Rue H, Martino S, Chopin N. Approximate Bayesian inference for latent Gaussian models by using integrated nested Laplace approximations. J R Stat Soc Series B Stat Methodol. 2009;71(2):319-92.

38. Spiegelhalter DJ, Best NG, Carlin BP, Van Der Linde A. Bayesian measures of model complexity and fit. J R Stat Soc Series B Stat Methodol. 2002;64(4):583-639.

39. Smith DL, Dushoff J, McKenzie FE. The risk of a mosquito-borne infectionin a heterogeneous environment. PLoS Biol. 2004;2(11):e368.

40. Thomas CJ, Cross DE, Bøgh C. Landscape movements of Anopheles gambiae malaria vector mosquitoes in rural Gambia. PLoS One. 2013;8(7):e68679.

41. Kirby MJ, Lindsay SW. Effect of temperature and inter-specific competition on the development and survival of Anopheles gambiae sensu stricto and Anopheles arabiensis larvae. Acta Trop. 2009;109(2):118-23.

42. World Health Organization. Indoor residual spraying: An operational manual for indoor residual spraying (IRS) for malaria transmission control and elimination. 2nd ed. Geneva: World Health Organization; 2015.

43. Jagannathan P, Muhindo MK, Kakuru A, Arinaitwe E, Greenhouse B, Tappero $J$, et al. Increasing incidence of malaria in children despite insecticidetreated bed nets and prompt anti-malarial therapy in Tororo, Uganda. Malar J. $2012 ; 11: 435$

44. Killeen GF, Seyoum A, Sikaala C, Zomboko AS, Gimnig JE, Govella NJ, White MT. Eliminating malaria vectors. Parasit Vectors. 2013;6:172.

45. Tangena J-AA, Adiamoh M, D'Alessandro $U$, Jarju $L$, Jawara $M$, Jeffries $D$, et al. Alternative treatments for indoor residual spraying for malaria control in a village with pyrethroid- and DDT-resistant vectors in the Gambia. PLoS One. 2013:8(9):e74351.

46. Cisse MBM, Keita C, Dicko A, Dengela D, Coleman J, Lucas B, et al Characterizing the insecticide resistance of Anopheles gambiae in Mali. Malar J. 2015;14(1):1-10.

47. Steinhardt LC, Yeka A, Nasr S, Wiegand RE, Rubahika D, Sserwanga A, et al. The effect of indoor residual spraying on malaria and anemia in a hightransmission area of northern Uganda. Am J Trop Med Hyg. 2013;88(5):855-61.

48. Kigozi R, Baxi SM, Gasasira A, Sserwanga A, Kakeeto S, Nasr S, et al. Indoor residual spraying of insecticide and malaria morbidity in a high transmission intensity area of Uganda. PLoS One. 2012;7(8):e42857.

49. Akogbeto M, Padonou GG, Bankole HS, Gazard DK, Gbedjissi GL. Dramatic decrease in malaria transmission after large-scale indoor residual spraying with bendiocarb in Benin, an area of high resistance of Anopheles gambiae to pyrethroids. Am J Trop Med Hyg. 2011;85(4):586-93.

50. Ahoua Alou LP, Koffi AA, Adja MA, Tia E, Kouassi PK, Koné M, Chandre F. Distribution of ace-1 $R$ and resistance to carbamates and organophosphates in Anopheles gambiae s.s. populations from Côte d'Ivoire. Malar J. 2010;9(1):1-7. 
51. Djogbénou L, Labbé $P$, Chandre F, Pasteur N, Weill M. Ace-1 duplication in Anopheles gambiae: a challenge for malaria control. Malar J. 2009;8(1):1-6.

52. World Health Organization. Global plan for insecticide resistance management in malaria vectors. Geneva: World Health Organization; 2012

53. Weiss D, Mappin B, Dalrymple U, Bhatt S, Cameron E, Hay S, Gething P. Reexamining environmental correlates of Plasmodium falciparum malaria endemicity: a data-intensive variable selection approach. Malar J. 2015;14(1):68.

54. Ingebrigtsen R, Lindgren F, Steinsland I. Spatial models with explanatory variables in the dependence structure. Spat Stat. 2014;8:20-38.

55. Lawson AB, Carroll R, Castro M. Joint spatial Bayesian modeling for studies combining longitudinal and cross-sectional data. Stat Methods Med Res. 2014;23(6):611-24.

56. Service MW. Mosquito (Diptera: Culicidae) dispersal - the long and short of it. J Med Entomol. 1997;34(6):579-88.

57. Pinder M, Conteh L, Jeffries D, Jones C, Knudsen J, Kandeh B, et al. The RooPfs study to assess whether improved housing provides additional protection against clinical malaria over current best practice in The Gambia: study protocol for a randomized controlled study and ancillary studies. Trials. 2016;17(1):275.

\section{Submit your next manuscript to BioMed Central} and we will help you at every step:

- We accept pre-submission inquiries

- Our selector tool helps you to find the most relevant journal

- We provide round the clock customer support

- Convenient online submission

- Thorough peer review

- Inclusion in PubMed and all major indexing services

- Maximum visibility for your research

Submit your manuscript at www.biomedcentral.com/submit 